\title{
DEPÓSITOS DE PERFLUOROCTANO EN USO \\ PROLONGADO COMO SUSTITUTIVO VÍTREO POSTQUIRÚRGICO
}

\section{DEPOSITS OF PERFLUOROCTANE AFTER PROLONGED USE AS A POST-OPERATIVE VITREOUS SUBSTITUTE}

\author{
ASENSIO-SÁNCHEZ VM ${ }^{1}$, TORREBLANCA-AGÜERA B ${ }^{2}$, MARTÍNEZ-CALVO S ${ }^{2}$, CALVO MJ ${ }^{2}$, \\ RODRÍGUEZ R ${ }^{1}$
}

\section{RESUMEN}

Caso clínico: En este trabajo se describe una paciente de 72 años con un desprendimiento de retina tratado con vitrectomía por pars plana (VPP) y como tamponador intraocular postquirúrgico una mezcla de aceite de silicona y perfluoro-n-octano. Tres meses después de la cirugía desarrolló sobre la retina inferior unos depósitos que correspondían a perfluoro-n-octano emulsificado.

Discusión: Depósitos de perfluoro-n-octano emulsificado con el aspecto de placas epiretinianas no se han descrito en la bibliografía como reacción adversa de los perfluorocarbonos.

Palabras clave: Desprendimiento de retina, vitrectomía pars plana, perfluorocarbono líquido, sustitutivo vítreo, depósitos epirretinianos.

\begin{abstract}
Case report: We describe a 72-year-old woman with a retinal detachment who underwent pars plana vitrectomy (PPV) and intraocular tamponade with a combination of silicone oil and perfluoro-noctane as a postoperative vitreous tamponade. Three months later, plaque-like deposits of emulsified perfluoro-n-octane were visible on the inferior retina.

Discussion: Deposits of emulsified perfluoro-noctane, as epiretinal plaques, have not been previously been described as adverse reactions following the use of perfluorocarbons in the eye (Arch Soc Esp Oftalmol 2007; 82: 241-244).
\end{abstract}

Key words: Retinal detachment, pars plana vitrectomy, perfluorocarbon liquid, vitreous substitute, epiretinal deposits.

\footnotetext{
Recibido: 26/1/06. Aceptado: 23/3/07.

Hospital General. Medina del Campo. Valladolid. España.

1 Doctor en Medicina.

2 Licenciado en Medicina.

Correspondencia:

V.M. Asensio Sánchez

Hospital General Servicio Castellano-Leonés de Salud

Servicio de Oftalmología

47010 Medina del Campo (Valladolid)

España

E-mail: vasensio@hmdc.sacyl.es
} 


\section{INTRODUCCIÓN}

En oftalmología la utilización de los perfluorocarbonos líquidos (PFCL) ha mejorado la cirugía retino-vítrea facilitando las maniobras quirúrgicas en los desprendimientos de retina complicados $(1,2)$. Los PFCL a pesar de ser considerados biológicamente inertes, no se recomiendan como sustitutivos vítreos por los efectos tóxicos que producen. Se describe un paciente en el que se utilizó perfluoroctano durante tres meses apareciendo unos depósitos epirretinianos en la retina en contacto con el PFCL.

\section{CASO CLÍNICO}

Mujer de 72 años, sin antecedentes familiares y oftalmológicos de interés, que acude a consulta externa enviada por su médico de atención primaria con el diagnóstico de catarata en el ojo derecho (OD). La agudeza visual en ese ojo era de percepción de luz, apreciándose un desprendimiento de retina total asociado a una vitreoretinopatía proliferante con múltiples agujeros en la retina periférica superior e inferior. En mayo de 2005 se realizó cirugía escleral (cerclaje) con VPP utilizando aceite de silicona 1000 cs $\left(\right.$ SIL-1000 ${ }^{\circledR}$. DORC, Zuidland, Holanda) con recidiva del desprendimiento de retina. En junio de 2005 se reopera utilizándose como tamponamiento temporal una mezcla de aceite de silicona 1000 cs y perfluoro-n-octano (Perfluoron ${ }^{\circledR}$.

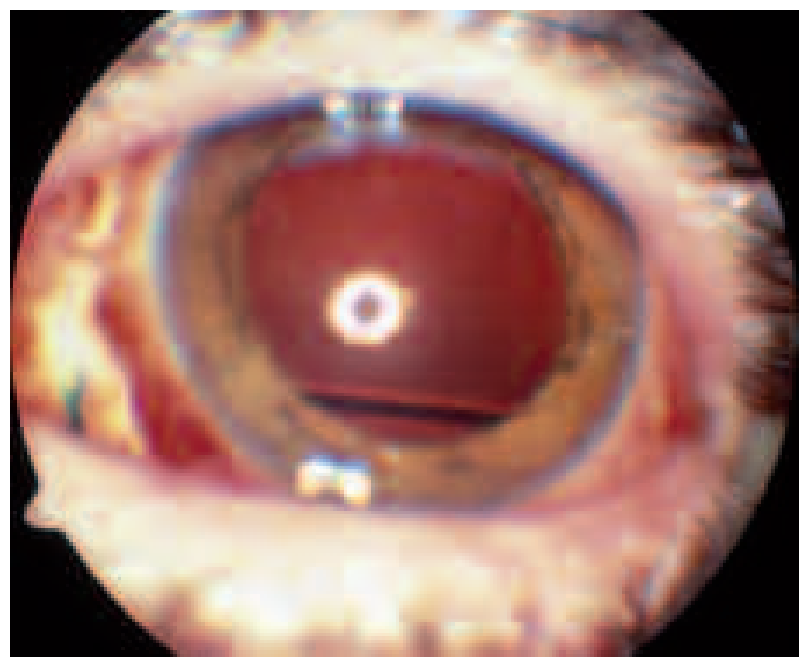

Fig. 1: Julio 2005. Evidente separación entre el PFCL y la silicona.
Alcon, Fort Worth, TX) consiguiéndose la reaplicación retiniana. En julio de 2005 la agudeza visual máxima en ese ojo era de 0,05 la retina estaba reaplicada y había una perfecta separación entre las dos substancias (fig. 1). En septiembre de 2005 una catarata nuclear impedía la visualización de la retina por lo que se realizó una vitrectomía pars plana combinada, para extraer la catarata y el tamponador. En la retina inferior había unos depósitos blanquecinos en forma de placas reticulares que con cromatografía líquida de alta resolución-espectrometría de masa se determinó que correspondían a PFCL emulsificado (fig. 2). En diciembre de 2005 la agudeza visual era de 0,05 con tensión intraocular de $12 \mathrm{mmHg}$ permaneciendo la retina reaplicada.

\section{DISCUSIÓN}

El PFCL llena el ojo en dirección póstero-anterior cuando se inyecta sobre el nervio óptico, tamponando la retina contra el epitelio pigmentario. $\mathrm{Su}$ alta gravedad específica junto a su inmiscibilidad con la sangre o el agua facilita la cirugía del desprendimiento de retina complicado y al tener una viscosidad entre 0,8 y $8,0 \mathrm{cs}$ a $25^{\circ} \mathrm{C}$ se facilitan las maniobras de inyección y extracción $(1,2)$. En este trabajo se describe una paciente con desprendimiento de retina complicado en la que se utilizó como tamponador postquirúrgico una mezcla de PFCL y aceite de silicona durante tres meses. Después de este tiempo se observaron depósitos blan-

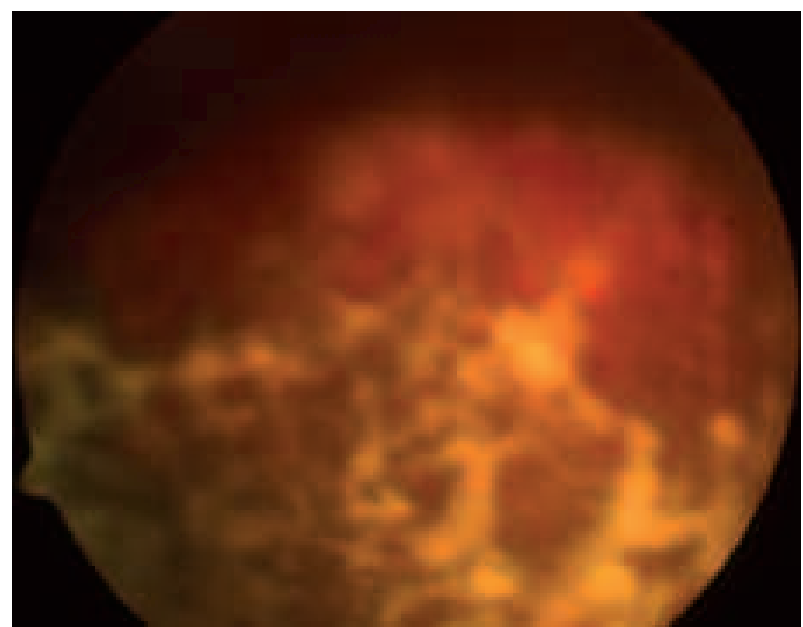

Fig. 2: Depósitos de PFCL que cubren la retina inferior (zona de contacto con el PFCL). La retina superior en contacto con la silicona está libre de depósitos. 
co-amarillentos en forma de placa en toda la retina inferior. El análisis bioquímico determinó que los depósitos eran perfluoro-n-octano emulsificado. No hemos encontrado ninguna referencia de depósitos epiretinianos en la revisión realizada en las bases de datos bibliográficas. Viebahn et al (3) utilizando perfluorofenantreno como sustitutivo vítreo durante 5 meses en un desprendimiento de retina del pseudofáquico, describieron depósitos blanquecinos sobre la lente de cámara anterior y el endotelio corneal que eran perfluorofenantreno emulsificado. Peyman et al (2) en una amplia revisión sobre los PFCL no hacen referencia a depósitos retinianos. Blinder et al (4) utilizaron perfluorofenantreno como sustitutivo vítreo postquirúrgico en 16 pacientes durante un máximo de 4 semanas, pero en ninguno de ellos refieren un cuadro retiniano como el que se describe en este trabajo. Mate y col (5) en un estudio experimental en ojos afáquicos y vitrectomizados demostraron desestructuración retiniana predominantemente en capas internas.
Los efectos secundarios de los PFCL, a nivel experimental y en los cuadros clínicos descritos, no los hace candidatos como tamponador postquirúrgico.

\section{BIBLIOGRAFÍA}

1. Chang S. Low viscosity liquid fluorochemicals in vitreous surgery. Am J Ophthalmol 1987; 103: 38-43.

2. Peyman GA, Schulman JA, Sullivan B. Perfluorocarbon liquids in ophthalmology. Surv Ophthalmol 1995; 39 : 375-395.

3. Viebahn M, Buettner H. Perfluorophenanthrene unsuitable for postoperative retinal tamponade. Am J Ophthalmol 1994; 118: 124-126.

4. Blinder KJ, Peyman GA, Desai UR, Nelson NC Jr, Alturki $W$, Paris $C L$. Vitreon, a short-term vitreoretinal tamponade. Br J Ophthalmol 1992; 76: 525-528.

5. Mate LA, López MI, Alonso I, Pastor JC, Alberola A, Sañudo MC. Tolerancia a largo plazo (3 meses) de la perfluorodecalina y la perfluorotributilamina. Análisis experimental. Arch Soc Esp Oftal Invest 1989; 2: 155-160. 